\title{
Merging and Arbitration Strategy Applied Bayesian Classification for Eye Location
}

\author{
Eun Jin Koh and Phill Kyu Rhee \\ Dept. of Computer Science \& Engineering Inha University \\ Biometrics Engineering Research Center \\ Yong-Hyun Dong, Incheon, Korea \\ supaguri@im.inha.ac.kr, pkrhee@inha.ac.kr
}

\begin{abstract}
Based on template facial features and image segmentation, this paper demonstrates a novel method for automatic detection of eyes in grayscale still images. A decision model of eye location is instituted by the priori knowledge of template facial features. After roughly detection of face, we apply three steps for system to locate eyes. Firstly, the Bayesian eye detector is used to find eye patterns in the upper region of the face image. This vector based Bayesian classifier adopts Haar transform as vectorize because we know that is robust at illumination variation. Secondly, merging and arbitration strategy are applied. It can manage variations of around eye regions due to spectacle rims or eye brows. Finally, Gaussian-projection function can locate robust precision eye position. The experimental results show that the proposed method can achieve higher performance at any test data.
\end{abstract}

\section{Introduction}

Constructing automatic face recognition system has been a promising field of computer vision and pattern recognition. The face recognition task is achieved in three steps, i.e. 1) face detection, 2) marking facial feature points and 3) face recognition.

The face detection determines whether or not there are any faces in the image and, if exist; notify the face location and range of each face. The face recognition identifies or authenticates one or more persons in the detected image using a stored database of faces. In general, face recognition system needs those remarkable facial landmarks such as eyes must be located before any other processing is performed. Since recognition algorithm is based on template matching, face must be accurately aligned before other recognition processing, which is usually achieved based on the location of eyes. Because marking facial feature is necessary step to the template matching, it is an important step to face recognition and eyes are crucial points of facial features. Therefore, automatically locating eyes is very significant stage.

There is a series of techniques that can effectively detect eyes in frontal upright face images. But they suffer from bad light conditions and a rim of glasses. As a matter of fact, eyebrows or thick glasses frame enough to be confused with eye that the classifier often makes a incorrect decision.

To detect eyes in rough face image which is detected by face detector, the size window is applied to every pixel position in the image. To detect eyes which are larger than the window size, size of input image is repeatedly reduced by method of 
super sampling or b-spline with factor 1.2 for each step, and the window is applied at each size. We define this method as multi-resolution. The multi-resolution method has some invariance at position and scale. The amount of invariance determines the number of windows which is must be applied at one image and it directly influences time of computation.

This paper introduces methodology for developed eye location procedure and depicts on both learning and estimating components. The experimental results are gained using the standard BioID, FERET and INHADB facial image database.

Detailed explanation of training images collection and methods are given in Section 2. The classifier architecture, postprocessing and arbitration strategy are given in Section 3. In Section 4, the experimentation of the system is described. Conclusions and directions for future research are presented in Section 5.

\section{Bayesian Discriminant Training Method}

In General, classification is the issue of predicting the class of a sample with probabilistic theories. The standard Bayesian algorithm produces a posterior probability - the probability of the class given that feature value has been measured.

Let $\omega_{1}$ and $\omega_{2}$ be the finite set of classes, and let $x$ be the feature vector of eye. Assume that $x$ is a d-dimension component vector and let be the conditional probability density function for $x$ with the probability density function for $x$ conditioned on being the true class. And let be the prior probability that class is, then the posterior probability can be computed from by Bayes formula. The extraction of features and training are learned supervised method.

To train the classifier a large number of eye and non-eye images are needed. 1,970 eye images were collected from face databases at FERET probe. The images include eyes of similar sizes, orientations, positions, and various intensities. These samples were used to normalize each eye to the same scale at pixels. These samples are converted to vectors and measured Mahalanobis distance using Bayesian discriminant method and PCA. In paper [5], to make vector of face they use 1D Haar wavelet representation, but we use $2 \mathrm{D}$ Haar wavelet representation. Because an eye image is 2D data, 2D Haar more suitable for representing features of eye than 1D Haar. To classify eye and non-eye using Bayesian method, at least two models [5] are required. One is eye class model and the other is non-eye class model.

In order to classify eye image from non-eye images, we need notion of distance. We refer to Mahalanobis distance instead of general Euclidean distance. It is useful measure of determining similarity of a test image set to a known one. It is different from Euclidean distance in that it takes into compute the correlations of the data set. Formally, the Mahalanobis distance from a group of values with mean $m=\left(m_{1}, m_{2}, m_{3}, \ldots, m_{n}\right)$ and covariance matrix $\sum$ for a multivariate vector is defined:

$$
d(x)=\sqrt{(x-m)^{t} \sum^{-1}(x-m)}
$$




\section{The Eye Location Method Under Bayesian Discriminant Framework}

The block diagram of the proposed method is shown in Fig. 1. When a rough face region is presented to the system, apply preprocessing to face image. Then creating vector and getting Mahalanobis distance are applied to each window. The detected positions which are classified as eye according to their distance are stored. The merging and arbitration strategy applied to outputs which are gathered from advance operation. In particular case, merging applied regions can be arbitrated. Because we know that one face image contains exactly two eyes, finally accepted regions are restricted in two.

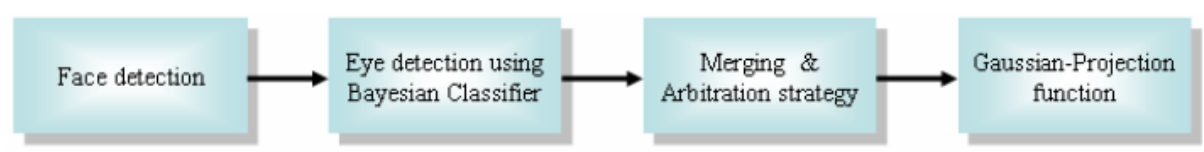

Fig. 1. Block diagram of the proposed eye location method.

\subsection{Eye location Using Bayesian Discriminant Method with Mahalanobis Distance}

We get Mahalanobis distance for each window. Suppose $d(x)_{e}$ is Mahalanobis distance for eye class, $d(x)_{n}$ is Mahalanobis distance for non-eye class. $d(x)_{e}$ and $d(x)_{n}$ can be calculated from the input pattern $x$, the eye class parameters (the mean eye, the covariance matrix), and the non-eye class parameters (the mean non-eye, the covariance matrix). We use two thresholds, $\theta$ and $\tau$ as follows:

$$
\begin{array}{ll}
\theta=\max \left(d(\alpha)_{e}\right) & \text { for sample } \alpha \text { that make }- \text { up of eye class } \\
\tau=\max \left(d(\beta)_{e}-d(x)_{n}\right) & \text { for sample } \beta \text { make - up of non-eye class }
\end{array}
$$

These are constant values, which are calculated when training time. Bayesian classifier offer classifying rule to the eye detection system, such that,

$$
x \in \begin{cases}\omega_{e} & \text { if }\left(d(x)_{e}<\theta\right) \text { and }\left(d(x)_{e}-d(x)_{n}<\tau\right) \\ \omega_{n} & \text { otherwise }\end{cases}
$$

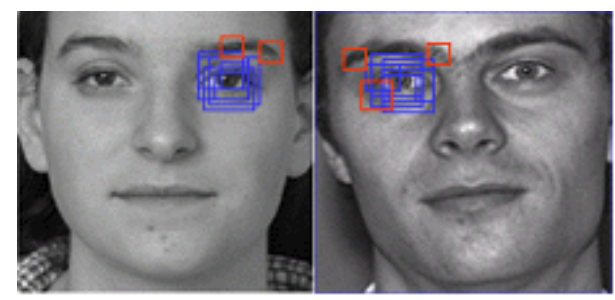

Fig. 2. Examples of detection 
Detected some examples are shown in Fig. 2. In the figure, each box represents the location and size of a window to which the Bayesian discriminant method gives a positive response. The blue box is correct location and the red box is false location. The Bayesian classifier has some invariance to position and scale, which results in multiple boxes around some faces. Note also that there are some false locations; they will be eliminated by methods presented in Section 3.3 and 3.4.

\subsection{Merging Strategy}

The examples in Fig. 2 showed that the raw output from Bayesian classifier may contain some false detection. In this section, we present one strategy to improve the credibility of location: merging overlapped detections [4] from Bayesian classifier.

There are many detected rectangles at multiple nearby eyes, while false detections usually arise with less frequency. This discovery gives us a heuristic that can eliminate much false detection. For each position and scale, the number of detected window within bounds of a specified neighborhood of that position can be counted. If the number is greater than specific number, the position is classified as an eye. The result position is indicated by the centroid of the neighbor detections, therefore duplicated detections can be eliminated. This method is operated as following manner:

a) The detections are recorded.

b) The centers of windows are calculated.

c) The centers are "spread out" and a threshold is applied.

d) The centroid of windows which satisfy threshold in scale and position are computed, and the centers of windows are collapsed to single point.

e) If another window overlaps the rectangle, the window is eliminated because it regarded as false detection.

This method is good at not only improving accept rate but decreasing false detection. If a specific position is correctly classified as an eye, then all other detected positions which overlap it are regarded as errors, therefore these can be eliminated. The position with the higher number of detections is conserved, and the position with the lower detections is eliminated. This method is affected by two variances that are threshold and size of spread out. Only accept a detection if there are at least threshold detections within a region (spread out along $x, y$, and scale) in the detections. The size of the region is determined by size, which is the number of pixels from the center of the region to its edge. We need to decide reasonable threshold and size for improve performance of system. Performance of eye location at 42 randomly extracted images

Table 1. Performance of eye location according to threshold and size

\begin{tabular}{c|c|c|c|c|c|c|c|c} 
Data Set & $\begin{array}{c}\text { merging } \\
(2,2)\end{array}$ & $\begin{array}{c}\text { merging } \\
(2,4)\end{array}$ & $\begin{array}{c}\text { merging } \\
(4,2)\end{array}$ & $\begin{array}{c}\text { merging } \\
(4,4)\end{array}$ & $\begin{array}{c}\text { merging } \\
(6,2)\end{array}$ & $\begin{array}{c}\text { merging } \\
(6,4)\end{array}$ & $\begin{array}{c}\text { merging } \\
(8,2)\end{array}$ & $\begin{array}{c}\text { merging } \\
(8,4)\end{array}$ \\
\hline FERET & $64.29 \%$ & $69.05 \%$ & $95.24 \%$ & $97.62 \%$ & $76.19 \%$ & $78.57 \%$ & $76.19 \%$ & $76.19 \%$ \\
\hline BioID & $69.05 \%$ & $64.29 \%$ & $95.24 \%$ & $95.24 \%$ & $76.19 \%$ & $76.19 \%$ & $73.81 \%$ & $78.57 \%$ \\
\hline \hline
\end{tabular}

merging (size, threshold): Only accept a eye candidate window if there are at least threshold detections within a square (spread out along $x, y$ ). The area of the square is determined by size, which is the distance from the center of the square to its edge. 
among each dataset according to threshold and size is shown in Table 1. And examples about this are shown in Fig. 3.

From the Table, we can see that the system generates highest accept rate when size is 4 and threshold is 4 . Therefore, we apply this value to the system.

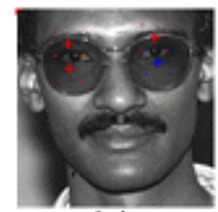

(a)

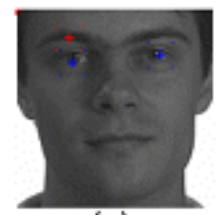

(e)

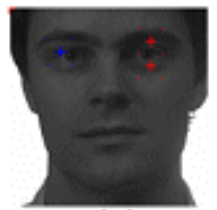

(b)

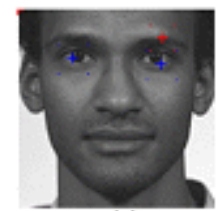

(f)

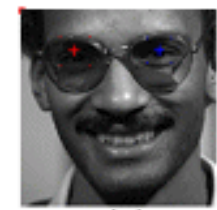

(c)

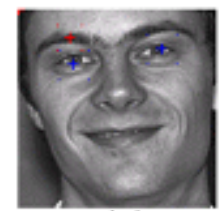

(g)

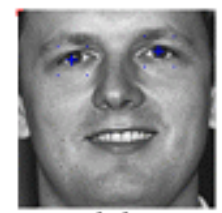

(d)

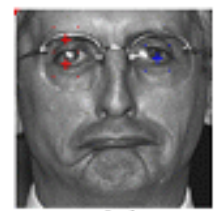

(h)

Fig. 3. Detection examples according to variable values

\subsection{Arbitration Strategy}

Sometimes even though merging is applied, two or more detected windows which gather around one eye are remained. As a matter of fact, eyebrows or thick rims of spectacle often look so similar to closed eyes that the classifier often makes a wrong decision. So both the eyes and neighborhood of eyes should be considered. Merging operates to remain region which has high detection density and to eliminate overlap windows. But if two or more high density regions exist independently around one eye, merging may product wrong result. Some example of errors is shown in Fig. 3. Red marks of (a), (b) and (h) are representative incorrect outputs via merging. These false outcomes are occurred by reason of peculiarity of their density distribution of windows which has produced by Bayesian discriminant classifier. Bayesian discriminant classifier often misclassifies eyebrows as eyes. Because the feature of eyebrow is similar to feature of eye, many detected rectangles are occurred around eyebrow. Since the positions with the higher number of detections are conserved at merging step, if density of detections at eyebrow is higher than that of eye, detections around eyebrow are reserved, but on the other hand detections around eye-center are eliminated by merging. In Fig. 4, (a) is detection around eyebrow which has highest density, (b) is detection around eye-center which has lower density than (a). Because the detection (b) overlap the detection (a) and density of (a) is higher than (b), detection (b) is eliminated by merging. Therefore, detection (d) and (e) are conserved only. In Fig. 5 we can see that detection (d) is upper region of eye-center and detection (f) is lower region of eye-center. A point (g) is centroid of detection (d) and (f). The point (g) is very close to center of eye. This heuristic method is called "Arbitration strategy". We showed case of arbitration among two error detections only, but Arbitration strategy is work under the situation that three or more error 
detections exist. Because we know that only one eye is in existence at left or right region of image, if there are two or more detections at left or right region, the system can derive correct location of eye by using Arbitration strategy.
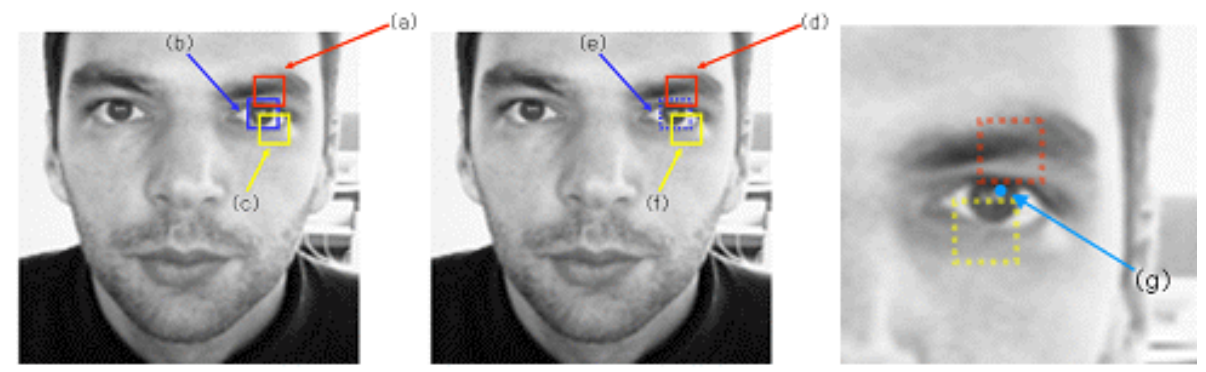

Fig. 4. Example of error result of merging. (a) is detection around eyebrow which has highest density, (b) is detection around eye-center, (c) is detection around eye which has lower density than (b). Because density of (a) is higher than density of (b), detection (b) is eliminated as (e). Therefore, finally detection (d) and (f) can be conserved only. A point ( $g$ ) is centroid of detection (d) and (f). The point ( $\mathrm{g}$ ) is very close to center of eye.

\section{Experimental Results}

\subsection{Data Sets}

The training set is obtained from FERET database, and totally 1970 eyes of 985 faces are extracted and normalized for training. Experimental test set consists of BioID (1521 images), FERET (3816 images), INHADB (1200 images), and totally 6537 faces are concerned in the evaluation of localization performance. The three test sets are from diverse sources to cover diverse eye variations in view angles, sizes, illumination, and glasses. Experiments based on such various sets should be able to test the generalization performance of our eye location algorithm.

\subsection{Evaluation Protocol}

To estimate the accuracy of eye location, a scale independent localization measure [3] is used. This relative error measure compares the eye automatic detected positions which are results of our system with the manually marked positions of each eye. $C_{l}$ and $C_{r}$ are the manually assigned left and right positions, $C_{l}^{\prime}$ and $C_{r}^{\prime}$ are the automatic detected position, $d_{l}$ is the Euclidean distance between $C_{l}^{\prime}$ and $C_{l}, d_{r}$ is the Euclidean distance between $C_{r}^{\prime}$ and $C_{r}, d_{l r}$ is the Euclidean distance between $d_{l}$ and $d_{r}$. The relative error of detection is defined as follows:

$$
e r r=\frac{\max \left(d_{l}, d_{r}\right)}{d_{l r}}
$$




\subsection{Comparison with Other Eye Location Methods}

Two different eye location methods are implemented and evaluated on the test set. Method 1: Method of detecting eyes using only Bayesian Classifier without arbitration strategy. Method 2: Algorithm of adding arbitration strategy step to Method1.

Table 2. Testing performance of Method $1($ err $<0.14)$

\begin{tabular}{|c|c|c|c|c|c|}
\hline data & source & images & accepted faces & false detects & accept rate \\
\hline \hline SET1 & FERET & 3816 & 3422 & 394 & $89.68 \%$ \\
\hline SET2 & BioID & 1521 & 1395 & 126 & $91.72 \%$ \\
\hline SET3 & INHADB & 1200 & 1090 & 110 & $90.83 \%$ \\
\hline Total & - & 6537 & 5907 & 630 & $90.36 \%$ \\
\hline
\end{tabular}

Table 3. Testing performance of Method $2($ err $<0.14)$

\begin{tabular}{|c|c|c|c|c|c|}
\hline data & source & images & accepted faces & false detects & accept rate \\
\hline \hline SET1 & FERET & 3816 & 3646 & 170 & $95.55 \%$ \\
\hline SET2 & BioID & 1521 & 1428 & 93 & $93.89 \%$ \\
\hline SET3 & INHADB & 1200 & 1132 & 68 & $94.33 \%$ \\
\hline Total & - & 6537 & 6206 & 331 & $94.94 \%$ \\
\hline
\end{tabular}

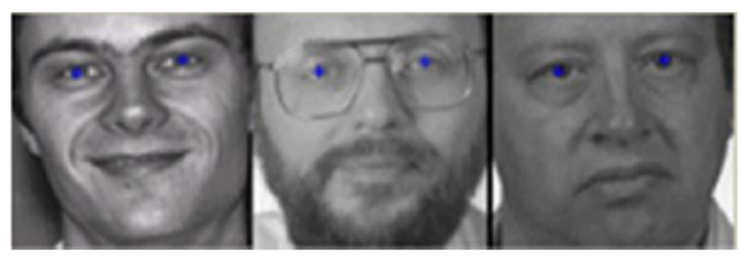

Fig. 5. Some eye location results from test sets

Performance of tow methods under err $<0.14$ are shown in Table 1 and Table 2. From the tables, we can see that Method 2 is better than Method 1. So we can conclude that arbitration strategy is effective for eye location. In addition, the average processing time per face of method 2 on an AMD Barton 2500+ PC system is $50 \mathrm{~ms}$ without code optimization. We show some outputs for visual inspection at Fig. 5.

Method 2 is compared with other systems. In paper [3], a detection rate is $99.1 \%$ under err $<0.2$. On the other hand, our detection rate is $99.31 \%$ when err $<0.2$ at all test sets. In paper [2], a detection is considered to be correct if err $<0.25$. Their detection rate on BioID dataset is $94.81 \%$. We evaluate method 2 on BioID under the same evaluation protocol. The detection rate of our system is $95.20 \%$ under err < 0.14 , and the detection rate is $97.63 \%$ under err $<0.25$. And their system achieve on $97.18 \%$ of JAFFE data set. But backgrounds and illumination conditions of JAFFE are not as complex and diverse as these of BioID. 


\section{Conclusion}

This paper describes a novel arbitration strategy applied Bayesian classifier for eye location. The system, which is trained on images from only a portion of one database, yet works on test images from diverse sources, displays robust generalization performance. The novelty of this paper comes from the combination of the $2 \mathrm{D}$ Haar based Bayesian classifier, the statistical modeling of eye and non-eye classes, and the arbitration strategy for growing performance. The arbitration strategy applied Bayesian classifier is trained with 1511 eye images and 3100 random natural (noneye) images. Experimental results using 6537 images (containing a total of 13074 eyes) from various image sources. The novel system achieves 94.94 percent eye detection accuracy under err $<0.14$.

In addition, because the arbitration strategy and Bayesian discriminant method don't localized for eye, it can be totally applied for location of other face organs such as nose or mouth.

\section{References}

[1] O. Jesorsky, K. Kirchberg, R. Frischholz, "Robust face detection using the Hausdorff distance," In: J. Bigun, F. Smeraldi Eds. Lecture Notes in Computer Science 2091, Berlin: Springer, 2001, pp.90-95.

[2] H. Zhou, X. Geng, "Projection functions for eye detection," Pattern Recognition, 2004, in press.

[3] Y. Ma, X. Ding, Z. Wang, N. Wang, "Robust precise eye location under probabilistic framework", IEEE International Conference on Automatic Face and Gesture Recognition, 2004

[4] Henry A. Rowley, Shumeet Baluja, Takeo Kanade, "Neural Network-Based Face Detection," IEEE Trans. Pattern Analysis and Machine Intelligence, vol. 20, NO. 1, 1998

[5] C. Liu, "A Bayesian Discriminating Features Method for Face Detection" IEEE Trans. Pattern Analysis and Machine Intelligence, vol. 25, no. 6, pp. 725-740, 2003

[6] C. Liu and H. Wechsler, "Gabor Feature Based Classification Using the Enhanced Fisher Linear Discriminant Model for Face Recognition," IEEE Trans. Image Processing, vol. 11, no. 4, pp. 467- 476, 2002.

[7] S.Lucey, S.Sridharan, V.Chandran, "Improved facial feature detection for AVSP via unsupervised clustering and dicriminant analysis", EURASIP Journal on Applied Signal Processing, vol 3, pp.264-275, 2003.

[8] T.Kawaguchi, D.Hikada, and M.Rizon, "Detection of the eyes from human faces by hough transform and separability filter," Proc. of ICIP, pp.49-52, 2000.

[9] P.Viola, M. Jones, "Rapid object detection using a Boosted cascade of simple features," Proc. of IEEE Conf. on CVPR, pp. 511 -518, 2001.

[10] Yongsheng Gao, Leung, "Face recognition using line edge map", Pattern Analysis and Machine Intelligence, IEEE Transactions on Volume 24, Issue 6, June 2002 Page(s):764 - 779

[11] J. Huang, X.H. Shao, H. Wechsler. "Pose Discrimination and Eye Detection Using Support Vector Machines", Proceeding of NATO-ASI on Face Recognition: From Theory to Applications, 1998.

[12] H.Schneiderman, T.Kanade. "A statistical model for 3D object detection applied to faces and cars," Proc. of IEEE Conf. on CVPR, 2000. 